\title{
Evaluation of drug-drug interactions and their clinical importance in a pediatric hematopoietic stem cell transplantation unit
}

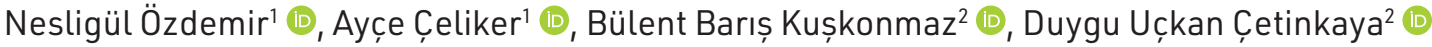 \\ 'Hacettepe University, Faculty of Pharmacy, Department of Clinical Pharmacy, Ankara, Turkey \\ ${ }^{2}$ Hacettepe University, Faculty of Medicine, Department of Pediatric Hematology, Ankara, Turkey
}

ORCID IDs of the authors: N.Ö. 0000-0003-2551-9549; A.C.. 0000-0001-6753-6844; B.B.K. 0000-0002-1207-4205; D.U.C. 0000-0003-3593-6493

Cite this article as: Ozdemir, N., Celiker, A., Kuskonmaz, B. B., \& Uckan, Cetinkaya, D. (2021). Evaluation of drug-drug interactions and their clinical importance in a pediatric hematopoietic stem cell transplantation unit. İstanbul Journal of Pharmacy, 51 (1), 1-7.

\begin{abstract}
Background and Aims: Many drugs with narrow therapeutic range and high toxicity risk are used in hematopoietic stem cell transplantation (HSCT) Units. The increase in the number of drugs raises the likelihood of interactions. This is particularly important in pediatric patients and may adversely affect the treatment process. In this study, we aimed to determine the potential drug interactions and to evaluate the clinical significance of them in terms of physician's and pharmacist's perceptions. Methods: The study was conducted as a prospective descriptive study over a six-month period in a tertiary care hospital's Pediatric HSCT Unit. A pharmacist evaluated inpatients' drugs for drug interactions by using a drug interaction checker program and the clinical significance of the interactions were evaluated by the physician and the pharmacist separately.

Results: Drugs used in 20 patients (median age $=8$ years, range $=0.6-17$ years) were evaluated. A total of 525 potential drugdrug interactions were identified. Two hundred and forty seven interactions (47.05\%) were major; 238 (45.33\%) were moderate; $23(4.38 \%)$ were contraindicated. The number of the interactions considered "clinically significant" by the pharmacist and "clinically insignificant" by the physician at the preparative regimen and post-transplant period were 15 (35.7\%) and 37 $(29.4 \%)$, respectively.

Conclusion: The management of drug interactions is important in pediatric HSCT patients as a vulnerable group. Drug interactions should be interpreted according to the patient's clinical presentation, not only theoretically. Cooperation between physicians and pharmacists in the management of interactions will contribute to optimize the patient's treatment.
\end{abstract}

Keywords: Drug interactions, pediatric patients, hematopoietic stem cell transplantation

\section{INTRODUCTION}

Drug interactions, one of the drug-related problems, are an important issue for both adult and pediatric patients. In particular, the intensive care unit, hematology, oncology, and hematopoietic stem cell transplantation (HSCT) are fields where multiple drugs are used and complex treatment strategies are required (Marcath, Coe, Hoylman, Redman, \& Hertz, 2018; Metzke et al., 2012; Aljadani \& Aseari, 2018; Rodrigues et al., 2017; Sanchez, Bacle, Lamy, \& Le-Corre, 2019). Many drugs with a narrow therapeutic index are used before and after transplantation. Thus drug interactions appear to be an important factor that may affect the success of the treatment (Trevisan, Silva, Oliveira, Secoli, \& Lima, 2015). Especially in patients who undergo allogeneic HSCT, which constitute a high-risk group, may develop clinically important drug interactions due to the large number of drugs used to prevent marrow rejection and immunological complications (Prot-Labarthe, Therrien, Demanche, Larocque, \& Bussieres, 2008). 
Preparative regimens including high-dose chemotherapy drugs, medications used for prophylaxis of veno-occlusive disease $(\mathrm{VOH})$, immunosuppressive drugs given to prevent the development of graft versus host disease (GVHD) after transplantation, and supportive therapies applied to prevent infections are related to the possible drug interactions and may affect the results of the treatment (Deeg, 2005). Alkylating drugs with narrow therapeutic index, especially busulfan and cyclophosphamide, which are included in the preparative regimens, interact with each other and with many other drugs due to their metabolization pathways (Myers et al., 2017). The blood level of cyclosporine, one of the drugs most frequently used for prophylaxis of GVHD, may vary due to interactions with many drugs such as azole antifungals, clarithromycin, phenobarbital, metronidazole, corticosteroids and etc. (Campana, Regazzi, Buggia, \& Molinaro, 1996; Sadaba, Lopez-deOcariz, Azanza, Quiroga, \& Cienfuegos, 1998). Supportive therapies to prevent complications include a large number of drug groups such as antimicrobials, antihypertensives, analgesics, mucosal protective drugs, anticoagulants, antiemetics and diuretics, and this situation possess a significant risk for drug interactions (Glotzbecker, Duncan, Alyea, Campbell, \& Soiffer, 2012).

The aim of this study is to determine the potential drug interactions of pediatric patients in a pediatric HSCT unit by a clinical pharmacist during transplantation periods and to evaluate the clinical significance of the interactions according to the physician's and the pharmacist's perceptions.

\section{MATERIALS AND METHODS}

The study was conducted as a prospective descriptive study between October 1 $1^{\text {st }}, 2015$ and May $1^{\text {st }} 2016$ in a tertiary care hospital's pediatric HSCT Unit inpatient service. The study was considered ethically appropriate with regards to GO 15 / 59604 dated 21.10.2015 by Hacettepe University Non-Interventional Clinical Research Ethics Committee.

Patients aged 0-18 years who were admitted to the HSCT unit for transplantation were included in the study. Informed consent was obtained from the patients and their relatives. The demographic data of the patients were collected from the patient files and the medication data were obtained from the hospital's electronic patient medication system by the clinical pharmacist. The potential interactions for each patient during the preparative regimen and post-transplantation periods were recorded daily. The clinical pharmacist participated in the physicians' morning rounds every day and examined and recorded every patient's drug orders before the visits. After the visits, every patient's drugs were checked in terms of potential drug interactions. If a new drug had been started at the weekend and/or out of working hours of the clinical pharmacist, the orders were examined retrospectively and detected interactions were recorded. This process continued until the patient was discharged. For every patient, the number of potential drug interactions was recorded but not calculated daily, it was calculated after the preparative regimen and the post-transplantation period had finished. For example, if there was a potential drug interaction during one or more days at any point of the transplantation period, it was calculated as "one potential drug interaction" at the end of the transplantation period. A current and scientific online database namely, Micromedex Solutions ${ }^{\circledR}$, which is one of the databases having high specificity, was used to detect drug interactions. The interactions in the Micromedex Solutions ${ }^{\oplus}$ database were categorized into four categories: "contraindicated, major, moderate, and minor". In this database contraindicated interaction means "The drugs are contraindicated for concurrent use". Major interaction means "The interaction may be life-threatening and/or require medical intervention to minimize or prevent serious adverse effects". Moderate interaction means "The interaction may result in exacerbation of the patient's condition and/or require an alteration in therapy". Minor interaction means "The interaction would have limited clinical effects; manifestations may include an increase in the frequency or severity of the side effects but generally would not require a major alteration in therapy". The clinical pharmacist verbally reported the identified interactions to the physician. In order to prevent an interaction, it was recommended to reduce the dose of the drug, to discontinue the drug or to select a drug that does not interact.

At the end of the study, a list showing the separate drug interactions detected in the preparative regimen and in the post-transplantation period was formed. When interactions that may lead to serious adverse events or adversely affect the treatment of HSCT occurred, they were classified as "clinically significant interactions". Interactions that have a tolerable adverse effect on the patient or that can be managed with drug dose adjustment or have an adverse effect that can be prevented by supportive treatments or is unlikely to occur are accepted as "clinically insignificant interactions". A physician with $>20$ years' experience and a pharmacist who was a clinical pharmacist in-training each examined the formed list and rated the drug interactions individually as mentioned above.

IBM SPSS $^{\circledR}$ version 22 program was used for statistical analysis of the data in the study. Distribution of data was identified by descriptive statistics (number and percentages). The number and the percentages of the interaction types and the clinically significant and clinically insignificant interactions were calculated.

\section{RESULTS}

During the study period, a total of 20 patients were followed up from hospital admission to discharge and potential drug interactions were detected. The patients' median age was 8 years (0.6-17 years). The demographic data of the patients are summarized in Table 1.

A total of 525 potential drug interactions were identified in 20 patients during the study. Two hundred and forty seven interactions (47.05\%) were major; 238 (45.33\%) were moderate; 23 (4.38\%) were contraindicated and 17 (3.24\%) were classified as minor according to the Micromedex Solutions ${ }^{\circledR}$ drug interaction checker program. It was found that $75.42 \%$ ( $n=396)$ of the potential interactions were caused by drugs 
Özdemir et al. Evaluation of drug-drug interactions and their clinical importance in a pediatric hematopoietic stem cell transplantation unit

Table 1. Demographic data of the patients.

\begin{tabular}{|c|c|}
\hline & n (\%) \\
\hline \multicolumn{2}{|l|}{ Gender } \\
\hline Female & $4(20.0)$ \\
\hline Male & $16(80.0)$ \\
\hline Total & $20(100.0)$ \\
\hline \multicolumn{2}{|l|}{ Diagnosis } \\
\hline ALL & $5(25.0)$ \\
\hline Thalassemia major & $3(15.0)$ \\
\hline WAS & $2(10.0)$ \\
\hline Fanconi anemia & $3(15.0)$ \\
\hline Others* & $7(35.0)$ \\
\hline \multicolumn{2}{|l|}{ Type of transplantation } \\
\hline Allogeneic & $19(95.0)$ \\
\hline Autologous & $1(5.0)$ \\
\hline \multicolumn{2}{|l|}{ Stem cell source } \\
\hline Peripheral blood stem cell & $4(20.0)$ \\
\hline Bone marrow & $16(80.0)$ \\
\hline \multicolumn{2}{|l|}{ Type of Preparative regimes } \\
\hline Myeloablative & $16(80.0)$ \\
\hline Reduced intensity/nonmyeloablative & $4(20.0)$ \\
\hline \multicolumn{2}{|c|}{$\begin{array}{l}\text { ALL; Acute Lymphoblastic Leukemia, WAS; Wiskott Aldrich Syndrome. } \\
\text { Others*: osteopetrosis, neuroblastoma, congenital neutropenia, } \\
\text { adrenoleuchodystrophy, LRBA (lipopolysaccharide - responsive } \\
\text { beige - like anchor) gene defect - immunodeficiency and juvenile } \\
\text { myelomonocytic leukemia (JMML); There was one patient with each } \\
\text { diagnosis. }\end{array}$} \\
\hline
\end{tabular}

used for HSCT treatment protocols (chemotherapeutics, immunosuppressants, antimicrobial prophylaxis). The most observed potential drug interaction (in $75 \%$ of the patients) was cyclosporine-fluconazole (major interaction).

Table 2 summarizes the distributions of the potential drug interactions detected in the preparative regimen and post-transplantation periods separately. During the preparative regimen period, 42 different drug pairs showed a total of 144 potential drug interactions. After transplantation, 126 different drug pairs showed a total of 381 potential drug interactions. One hundred and thirty seven of 381 interactions detected after transplantation were due to cyclosporine interactions with 22 different drugs. Nine of them were major; 13 were moderate. The drugs with the highest interaction rate with cyclosporine were fluconazole, methylprednisolone (MPZ), methotrexate (MTX), metronidazole, amikacin, ciprofloxacin and furosemide, respectively.

The data of major interactions detected during the preparative regimen period are summarized in Figure 1. The moderate interactions are given in the Figure 2.

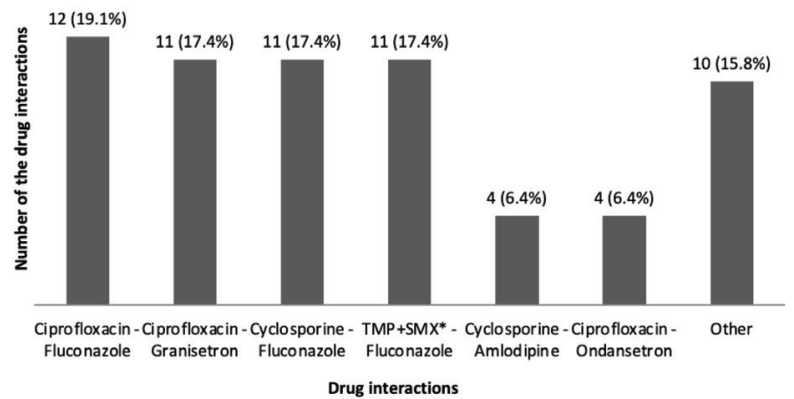

Figure 1. Distribution of major interactions detected at the preparative regimen period $(n=63)$.

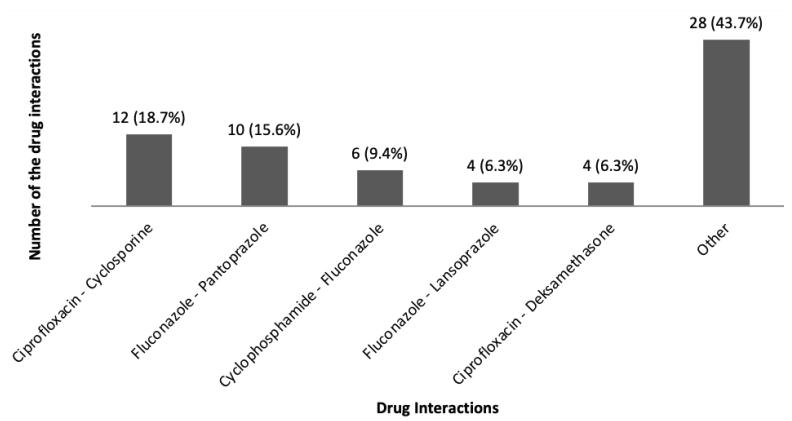

Figure 2. Distribution of moderate interactions detected at the preparative regimen period $(n=64)$.

Seventy five percent of the contraindicated interactions ( $n=$ 12) were fluconazole-granisetron; $18.7 \%(n=3)$ were fluconazole-ondansetron and $6.3 \%(n=1)$ were calcium gluconateceftriaxone interactions in the preparative regimen period. The only minor interaction detected during the preparative regime was the interaction between amikacin and piperacillin/tazobactam.

Table 2. Distribution of drug interactions detected at preparative regimen and post-transplantation period according to the interaction degree.

\begin{tabular}{|c|c|c|c|c|c|}
\hline & $\begin{array}{c}\text { Major } \\
\text { interaction }\end{array}$ & $\begin{array}{c}\text { Moderate } \\
\text { interaction }\end{array}$ & $\begin{array}{c}\text { Minor } \\
\text { interaction }\end{array}$ & $\begin{array}{l}\text { Contraindicated } \\
\text { interaction }\end{array}$ & Total \\
\hline & n (\%) & n (\%) & n (\%) & n (\%) & \\
\hline Preparative regimen period & $63(43.75)$ & $64(44.40)$ & $1(0.70)$ & $16(11.15)$ & 144 \\
\hline Post-transplantation period & $184(48.30)$ & $174(45.70)$ & $16(4.20)$ & $7(3.90)$ & 381 \\
\hline
\end{tabular}


The data of major interactions and moderate interactions detected at the post-transplantation period are summarized in Figures 3 and 4, respectively.

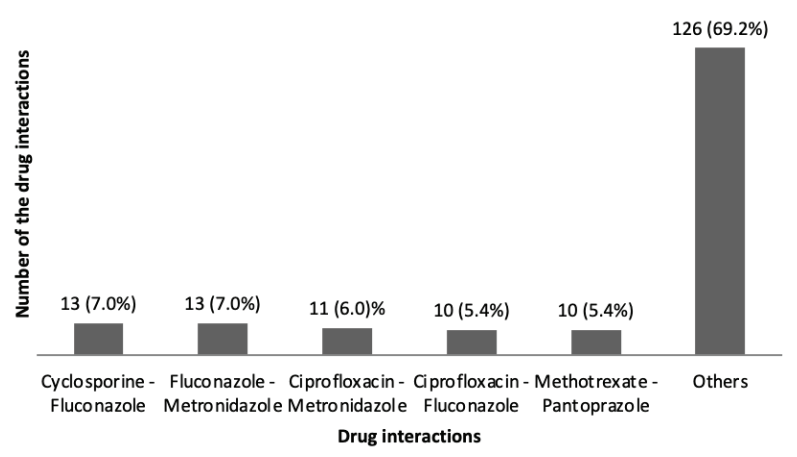

Figure 3. Distribution of the major interactions detected at the posttransplantation period $(n=184)$.

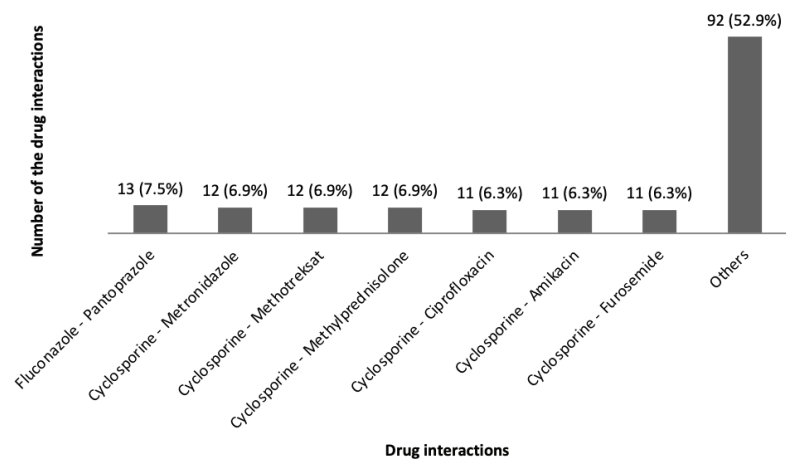

Figure 4. Distribution of the moderate interactions detected at the post-transplantation period $(n=174)$.

The number and percentages of contraindicated drug interactions detected at the post-transplantation period were fluconazole-granisetron; 3 (42.8\%), fluconazole-ondansetron; 3 (42.8\%) and clarithromycin-fluconazole; 1 (14.4\%).

The number and percentages of minor interactions detected at the post-transplantation period were amikacin-piperacillin/ tazobactam; 10 (58.89\%), folic acid-MTX; 2 (11.6\%), furosemidephenytoin; 1 (5.8\%), ferro glycol sulfate-sodium bicarbonate; 1 (5.8\%), clarithromycin-lansoprazole; 1 (5.89\%) and calcium carbonate-ferro glycol sulfate; 1 (5.8\%).

Table 3 summarizes the data of the drug interactions' clinical significance determined by the pharmacist and physician sep- arately. Forty-two drug interactions in the preparative regimen period and 126 drug interactions in the post-transplantation period were evaluated in terms of clinical significance. In the preparative regimen period, 15 (35.7\%) interactions were defined as "clinically significant" by the pharmacist, and as "clinically insignificant" by the physician. Two (4.8\%) interactions were considered as "clinically insignificant" by the pharmacist and as "Clinically significant" by the physician. While, 7 (16.6\%) interactions were considered as "clinically significant", 18 (42.9\%) interactions were considered as "clinically insignificant" by both clinical pharmacist and physician.

In the post-transplantation period 37 (29.4\%) interactions were defined as "clinically significant" by the pharmacist, and as "clinically insignificant" by the physician. Two (1.6\%) interactions were considered as "clinically insignificant" by the pharmacist and as "clinically significant" by the physician. While, 29 (23.0\%) interactions were considered as "clinically significant, 58 (46.0\%) interactions were considered as "clinically insignificant" by both the clinical pharmacist and the physician.

\section{DISCUSSION}

Many drugs are used for different purposes in HSCT. The majority of these drugs have a narrow therapeutic index and are highly toxic. The rise in the number of drugs taken by a patient increases the probability of interaction occurrence (Leather, 2004). Drug interactions are one of the major drugrelated problems that have the potential to adversely affect the treatment process in both adult and pediatric patients. There are a limited number of studies evaluating drug interactions in pediatric hematology-oncology and HSCT patients (Tavousi, Sadeghi, Darakhshandeh, \& Moghaddas, 2019; Balk et al., 2017). In these studies, only the interactions of the drugs used specifically for stem cell transplantation were evaluated, not all the drugs including the ones used for other indications (Valenzuela et al., 2017; Bernard, Goutelle, Bertrand, \& Bleyzac, 2014). Apart from HSCT specific drugs, the use of non-immunosuppressant and non-chemotherapeutic drugs are common in pediatric HSCT patients and these drugs may interact with each other or with the HSCT specific drugs (Eldesouky, Li, Abutaleb, Mohammad, \& Seleem, 2018). In Turkey, there have been no studies that evaluate these drug interactions in the pediatric HSCT patient population.

Trevisan et al. evaluated the prevalence of potential drug interactions of the drugs received on the day of transplantation by the HSCT patients (Trivesan et al., 2015). Interactions were ana-

Table 3. Distribution of the clinical importance of drug interactions detected during the pre-transplantation ( $n=42$ ) and post-transplantation period $(n=126)$ according to pharmacist and physician.

\begin{tabular}{|lcccc|}
\hline & \multicolumn{2}{c}{ Preparative regimen period } & \multicolumn{2}{c|}{ Post-transplantation period } \\
\cline { 2 - 5 } & $\begin{array}{c}\text { Clinically significant } \\
\text { interactions } \\
\mathbf{n}(\%)\end{array}$ & $\begin{array}{c}\text { Clinically insignificant } \\
\text { interactions } \\
\mathbf{n}(\%)\end{array}$ & $\begin{array}{c}\text { Clinically significant } \\
\text { interactions } \\
\mathbf{n}(\%)\end{array}$ & $\begin{array}{c}\text { Clinically insignificant } \\
\text { interactions } \\
\mathbf{n}(\%)\end{array}$ \\
\hline Clinical pharmacist & $22(52.3)$ & $20(47.7)$ & $66(52.3)$ & $60(47.6)$ \\
Physician & $9(21.4)$ & $33(78.6)$ & $31(24.6)$ & $95(75.4)$ \\
\hline
\end{tabular}


lyzed by an interaction program called DRUG-REAX ${ }^{\circledR}$. At least one clinically significant interaction was detected in $82.5 \%$ of 40 patients and $80.9 \%$ of the detected interactions were classified as major interactions.

In a retrospective study, Gholaminezhad et al. (2014) evaluated potential drug interactions before and after transplantation in adolescent and adult HSCT patients (Gholaminezhad et al., 2014). Drug interactions were classified by using the Lexi-Interact ${ }^{\circledast}$ interaction program. Minor interactions and intravenous drug incompatibilities were excluded; only moderate and major interactions were reported. All patients had at least one interaction and a total of 13,600 potential drug interactions were detected in 384 patients. Almost $82 \%$ of the interactions were ranked as moderate. The most common interaction was the interaction between trimethoprim/sulfamethoxazole and fluconazole. Granisetron-fluconazole and fluconazole-phenytoin interactions followed them. Sixty one point five percent of interactions were due to HSCT-related drugs (preparation regimen drugs, immunosuppressants and antimicrobials).

In the present study, 525 potential drug interactions were detected in 20 patients. Forty seven percent of the interactions were classified as major interaction and $45.33 \%$ were classified as moderate interaction. The number of major and moderate interactions were close to each other. This finding highlighted that the interactions occurring in pediatric HSCT patients may lead to more serious consequences if not prevented or controlled. In our study, the rate of HSCT specific drug interactions was lower (75.42\%) than Gholaminezhad et al. (2014)'s study. This finding was associated with the possibility of more drug use in adult patients. The most frequent interaction observed was between cyclosporine and fluconazole, fluconazole being incriminated in both studies. The widespread use of both drugs in HSCT and their high interaction potential may have contributed to this result.

In this study, of the 381 interactions detected after transplantation, 35.9\% were cyclosporine interactions with other drugs. Cyclosporine is frequently used in allogeneic transplantations other than autologous transplantations. Cyclosporine blood levels should be kept within the ideal range in order to prevent both stem cell rejection and the development of GVHD (Zeighami et al., 2014). The cyclosporine blood level may increase or decrease due to its interaction with other drugs. Therefore, strict monitoring of the blood level is necessary and adjusting of the dose of cyclosporine is important for the success of HSCT. The fact that cyclosporine was responsible for one third of the interactions detected in the post-transplantation period supports that all drugs used concurrently with cyclosporine should be evaluated carefully in terms of interaction.

The detected interactions were potential interactions and it was difficult to distinguish the clinical outcomes of them in practice. Some specific drug interaction effects, such as increase in liver transaminases (cyclosporine-caspofungin) and creatinine levels (cyclosporine-nephrotoxic drugs), were seen clinically.
The long and complex HSCT process and polypharmacy increase the probability of drug interactions. Prevention of interactions is often possible by selecting the drugs that do not interact, or removing one of the drugs that display interaction. Therefore, the interaction probability needs to be carefully evaluated. In order to guide the treatment process correctly, determining the clinical significance of potential drug interactions will be a valuable support for the physicians. All interactions detected through drug interaction checker programs may not be clinically important (Ament, Bertolino, \& Liszewski, 2000). In a study by Chan et al. (2009) in which the interactions between oral anticancer and non-cancer drugs were evaluated in terms of clinical significance, 41 different drug interactions were detected through the Drugdex ${ }^{\circledR}$ database (Chan, Tan, Wong, Yap, \& Ko, 2009). Nine pharmacists evaluated all the interactions and $17.1 \%$ of the interactions were not considered clinically important by all the pharmacists.

In our study, approximately half of the interactions detected during the preparation regimen and after the transplantation were found to be clinically important by the pharmacist. Unlike the studies in the literature, the proportion of clinically insignificant interactions was found to be higher. Firstly, only one clinical pharmacist's evaluation of the interactions may have led to this discrepancy. Secondly, while only anticancer drugs were evaluated in the study of Chan et al., all drugs' interactions were evaluated in our study. While anticancer drugs have a high potential of clinically significant interactions, drugs used for supportive treatment may have clinically less important interactions.

In this study, the pharmacist and physician's interpretations of clinical interactions were also compared. The rate of the clinically significant interactions, both in the pre- and post-transplantation periods, evaluated by the physician was lower than that of the pharmacist. In our study, concomitant use of cyclosporine with another drug that has a nephrotoxic effect was considered clinically significant interaction by the physicians. If the adverse event due to drug interaction can be controlled by supportive treatments or monitoring of the drug level, then, that interaction is considered as less clinically important by the physician. This indicates that physicians pay regard to the benefit/harm relationship in the treatment of critically important patients such as HSCT patients and take into account the interactions with only very serious results as a general approach.

The number of interactions determined as clinically significant was higher in the post transplantation period than the preparative regimen period for both pharmacist and physician evaluations. The post transplantation period is a critical and complex period because of engraftment expectation and possibility of GVHD. Therefore, it has a burden of polypharmacy. The drugs used after transplantation are the drugs that have a high possibility of adverse effect and drug interactions. Both the pharmacist and physician who consider these post transplantation drugs were more alert to prevent undesired effects of these drugs. As a consequence, the number of clinically significant interactions was found to be higher than that of the preparative regimen period that had a lower drug burden. 
Considering factors such as concomitant drug administration, drug administration site and age of the patient, the interaction as assumed "clinically insignificant" by the clinicians if it was unlikely to occur even if it was theoretically a major interaction or contraindicated. For example, calcium gluconate-ceftriaxone is an age specific (for neonates) contraindicated interaction but both pharmacist and physician determined it as "clinically insignificant" in the HSCT service, which had no neonate patients. Besides, the minor drug interactions (e.g., amikacin and piperacillin/tazobactam, clarithromycin and lansoprazole, furosemide and phenytoin) were determined "clinically insignificant" by both the pharmacist and physician. At this point, the importance and the difference of the drug interaction checker programs' theoretical data and the practice experience of the clinicians gained more value.

This study is the first one to evaluate drug interactions in pediatric HSCT patients in Turkey. Although there are lots of studies about drug interactions in adult patients, these studies cannot adequately guide the clinicians that work in the pediatric services due to pharmacokinetic differences between pediatric and adult subjects. Therefore, this study will be beneficial for clinicians to manage the treatment of pediatric HSCT patients when they encounter drug interactions.

This study's limitations are, the evaluation of the clinical significance of the interactions was performed by only one pharmacist and one physician, it was a short-term study and it had a limited number of patients for evaluation.

\section{CONCLUSION}

Drug interactions in areas where polypharmacy can be encountered frequently may be an obstacle for giving an optimal treatment regimen. Pediatric stem cell transplantation units are among the areas where polypharmacy is common. Although pediatric patients are exposed to less polypharmacy than adults, management of drug interactions is important due to pediatric patients vulnerability. Not only chemotherapeutic and immunosuppressant drugs but also other drugs used for antimicrobial prophylaxis and supportive therapies may have important interactions. Drug interaction databases are frequently used in clinical practice and guide clinicians for the regulation of treatments. However, the clinical significance of drug interactions detected in databases can be interpreted differently by the physicians and clinical pharmacists. Drug interactions should be evaluated according to the patient's clinical situation, not just as the theoretical information. The cooperation of physicians and clinical pharmacists will contribute to optimizing the treatment of the patient and the management of drug interactions.

Ethics Committee Approval: The study was considered ethically appropriate with regards to GO 15 / 596-04 dated 21.10.2015 by Hacettepe University Non-Interventional Clinical Research Ethics Committee.

Informed Consent: Written consent was obtained from the participants.

Peer-review: Externally peer-reviewed.
Author Contributions: Conception/Design of Study- N.Ö., A.Ç., D.U.Ç.; Data Acquisition- N.Ö., D.U.Ç.; Data Analysis/Interpretation- N.Ö., A.Ç., B.B.K., D.U.Ç.; Drafting Manuscript- N.Ö., A.Ç.; Critical Revision of Manuscript- A.Ç., B.B.K., D.U.Ç.; Final Approval and Accountability- N.Ö., A.Ç., B.B.K., D.U.Ç.; Technical or Material Support- D.U.Ç.; Supervision- A.Ç., B.B.K., D.U.Ç

Conflict of Interest: The authors have no conflict of interest to declare.

Financial Disclosure: Authors declared no financial support.

Acknowledgement: The authors would like to thank Assoc. (MD) Prof. Fatma Visal Okur, Coordinator of Nurses Nevin Cetin and Head Nurse Fatma Kırac for supporting clinical activities and Pharmacist Anna Maria Kuyumcu for English editing.

\section{REFERENCES}

- $\quad$ Aljadani, R., \& Aseeri, M. (2018). Prevalence of drug-drug interactions in geriatric patients at an ambulatory care pharmacy in a tertiary care teaching hospital. BMC Research Notes, 11(1). http:// dx.doi.org 10.1186/s13104-018-3342-5

Ament, P. W., Bertolino, J.G., \& Liszewski, J.L. (2000). Clinically significant drug interactions. American Family Physician, 61(6), 1745-1754.

- Balk, T. E., Van-der-Sijs, I. H., Van-Gelder, T., Janssen, J. J. B., Van-der Sluis IM, Van-Leeuwen, R. W. F., \& Engels, F. K. (2017). Drug-drug interactions in pediatric oncology patients. Pediatric Blood Cancer. 64(7). https://doi.org/10.1002/pbc.26410

- $\quad$ Bernard, E., Goutelle, S., Bertrand, Y., \& Bleyzac, N. (2014). Pharmacokinetic drug-drug interaction of calcium channel blockers with cyclosporine in hematopoietic stem cell transplant children. Annals of Pharmacotherapy, 48(12), 1580-1584. https://doi. org/ 10.1177/1060028014550644

- $\quad$ Campana, C., Regazzi, M. B, Buggia, I., \& Molinaro, M. (1996). Clinically significant drug interactions with cyclosporin - An update. Clinical Pharmacokinetics, 30(2), 141-179.

- Chan, A., Tan, S. H., Wong, C. M., Yap, K. Y., \& Ko, Y. (2009). Clinically significant drug-drug interactions between oral anticancer agents and nonanticancer agents: a Delphi survey of oncology pharmacists. Clinical Therapeutics, 31(2), 2379-2386. https://doi. org/10.1016/j.clinthera.2009.11.008

- Deeg, H. J. (2005). Optimization of transplant regimens for patients with myelodysplastic syndrome (MDS). Hematology American Society of Hematology Education Program, 167-173.

- Eldesouky, H. E., Li, X., Abutaleb, N. S., Mohammad, H., \& Seleem, M. N. (2018). Synergistic interactions of sulfamethoxazole and azole antifungal drugs against emerging multidrug-resistant Candida auris. International Journal of Antimicrobial Agents, 52(6), 754-761. https://doi.org/10.1016/j.ijantimicag.2018.08.016

- Gholaminezhad, S., Hadjibabaie, M., Gholami, K., Javadi, M. R., Radfar, M., Karimzadeh, I., \& Ghavamzadeh, A. (2014). Pattern and associated factors of potential drug-drug interactions in both preand early post-hematopoietic stem cell transplantation stages at a referral center in the Middle East. Annals of Hematology, 93(11), 1913-1922.

- Glotzbecker, B., Duncan, C., Alyea, E., Campbell, B., \& Soiffer, R. (2012). Important drug interactions in hematopoietic stem cell transplantation: what every physician should know. Biology of Blood and Marrow Transplantation, 18(7), 989-1006. https://doi. org/ 10.1016/j.bbmt.2011.11.029

- Leather, H. L. (2004). Drug interactions in the hematopoietic stem cell transplant (HSCT) recipient: what every transplanter needs to know. Bone Marrow Transplantation, 33(2), 137-152. 
- Marcath, L. A., Coe, T. D., Hoylman, E. K., Redman, B. G., \& Hertz, D. L. (2018). Prevalence of drug-drug interactions in oncology patients enrolled on National Clinical Trials Network oncology clinical trials. BMC Cancer, 18(1), 1155-1163. http://dx.doi.org/10.1186/ s12885-018-5076-0

- Metzke, B., Hug, M. J., Fink, G., Hieke, S., Jung, M., \& Engelhardt, M. (2012). Drug-drug interactions in the hematology and oncology department: a real-life assessment of frequency and severity. Blood, 120(21), 4250-4250. Retrieved from https://www.researchgate.net/publication

- Myers, A. L., Kawedia, J. D., Champlin, R. E., Kramer, M. A., Nieto, Y., Ghose, R., \& Andersson, B. S. (2017). Clarifying busulfan metabolism and drug interactions to support new therapeutic drug monitoring strategies: a comprehensive review. Expert Opinion on Drug Metabolism\& Toxicology, 13(9), 901-923. https://doi.org/ 10. 1080/17425255.2017.1360277

- $\quad$ Prot-Labarthe, S., Therrien, R., Demanche, C., Larocque, D., \& Bussieres, J. F. (2008). Pharmaceutical care in an inpatient pediatric hematopoietic stem cell transplant service. Journal of Oncology Pharmacy Practice, 14(3), 147-152. https://doi. org/ 10.1177/1078155208093929

- Rodrigues, A. T., Stahlschmidt, R., Granja, S., Pilger, D., Falcao, A. L. E., \& Mazzola, P. G. (2017). Prevalence of potential drug-drug interactions in the intensive care unit of a Brazilian teaching hospital. Brazilian Journal of Pharmaceutical Science, 53(1), e16109- e16117. https://doi.org/10.1590/s2175-97902017000116109

- Sadaba, B., Lopez-de-Ocariz, A., Azanza, J. R., Quiroga, J., \& Cienfuegos, J. A. (1998). Concurrent clarithromycin and cyclosporin A treatment. Journal of Antimicrobial Chemotherapy, 42(3), 393-395.
Sanchez, L., Bacle, A., Lamy, T., \& Le-Corre, P. (2019) Potential drugdrug interactions and nephrotoxicity in hematopoietic stem cell transplant adult recipients during bone marrow transplantation unit stay. Cancer Chemotherapy and Pharmacology, 83(5), 827835. https://doi.org/10.1007/s00280-019-03791-9.

- Tavousi, F., Sadeghi, A., Darakhshandeh, A., \& Moghaddas, A. (2019). Potential drug-drug interactions at a referral pediatric oncology ward in Iran: A cross-sectional study. Journal of Pediatric Hematology Oncology, 41(3), e146-e151. https://doi.org/10.1097/ MPH.0000000000001346

Trevisan, D. D., Silva, J. B., Oliveira, H. C., Secoli, S. R., \& Lima, M. H. (2015). Prevalence and clinical significance of potential drugdrug interaction in hematopoietic stem cell transplantation. Cancer Chemotherapy and Pharmacology, 75(2), 393-400. https://doi. org/10.1007/s00280-014-2657-8

- Valenzuela, R., Torres, J. P., Salas, C., Gajardo, I., Palma, J., Catalan, P. ... Morales, J. (2017). Drug interaction of voriconazole-cyclosporine in children undergoing hematopoietic stem cell transplantation (2013-2014). Revista Chilena de Infectologia, 34(1), 14-18. https://doi.org/ 10.4067/s0716-10182017000100002

- Zeighami, S., Hadjibabaie, M., Ashouri, A., Sarayani, A., Khoee, S. H., Mousavi, S. ... Ghavamzadeh, A. (2014). Assessment of cyclosporine serum concentrations on the incidence of acute graft versus host disease post hematopoietic stem cell transplantation. Iranian Journal of Pharmaceutical Research, 13(1), 305-312. 